\title{
Research on the Psychological Course and Development Trend of Vocal Performance
}

\author{
Zhang Ning \\ Academy of Music, Nanyang Institute of Technology, Nanyang, China \\ 47210605@qq.com
}

Keywords: Vocal art, Performance psychology, Vocal aesthetics

\begin{abstract}
The psychology of vocal music, which is separated from the art of vocal music, has already been formed as an independent discipline and guides the development of vocal music. The purpose of studying psychology of vocal music performance is to theoretically clarify the performers' psychological phenomena, essence and rule, and to promote the development of the art of vocal music by fully displaying the psychological factors' regulative function to performance and even the art of vocal music itself.
\end{abstract}

\section{Introduction}

In today's society, where psychology is widely valued, vocal art psychology, which has been branched out of vocal art, has been formed and guided by the development of vocal art as an independent discipline [1-2]. Vocal art performance psychology with its unique status and academic value, in the field of vocal music has been unprecedented development. The voice of singing is the reaction or manifestation of inner feelings, thus revealing the law of emotional psychology to control the sound [3]. Studying vocal art performance psychology should stand in the cross angle of vocal psychology and vocal music performance, in order to reveal the essence of vocal performance psychological phenomenon and the development of scientific laws [4]. Its purpose is to understand the psychological phenomenon, essence and law of the performer in theory, so as to improve and prosper the vocal art and adapt to the needs of the society by giving full play to the regulating effect of psychological factors on the performance and the whole vocal art itself [5-6].

\section{The Historical Echo of the Development of Vocal Music Performing Psychology}

In China, since the primitive society, singing has been fully practiced and developed. The rituals and prayer rituals, fishing and farming and other labor, and even love can not be separated from singing. The Book of Songs, the Songs of the South, Yuefu in later period, more or less revealed in the long social life singing the development and achievements, such as Xia Yu era "beggar Yi $\mathrm{Xi}$... ..." which sings a couple suspense. The ancients expressed or delighted in the form of singing, or sad, or sad, or kind, or evil. It shows that the sound of singing is the reaction or manifestation of the inner emotion, which reveals the regulation of the emotional psychology. When singing, although have not formed an independent vocal music art, but has become a way for people to express their emotions, some of the characteristics of performing psychological also gave birth to them. In ancient Greek and Christian cultures in Europe, it was also evident that singing was used in the marriage and sacrifice of People's Daily lives.

It wasn't until the 17th century that the importance of acting psychological problems caught the attention of many vocalists and was involved in all aspects of vocal psychology. Such as the act of singing with rod, Ms. Dupray of the singing art and the art of singing of Jacques Garcia each performance, directly or indirectly $r$ expounds the psychological point of view. Italian founder of vocality Enrico caruso in his "my experience of vocal music, there are many stories about the psychological performance, such as:" must be aware of their inner need to sing all the songs, can perfectly to all the people must have the deepest infections. Otherwise, his singing is no mood and appeal." In his book, he also sets chapters on the various strains of the singer and the performance of many singers. By the 19th century, the most outstanding vocal music professor in Italian vocal 
school, Lan PeiEr further studied the vocal music psychology problem, that association and imagination, talent, temperament, ability to feel is widely used in teaching, but also the cultivation of aesthetic ability on the agenda. Since then, vocal musician Hobart - Caesar, in his two books "song of science and feeling", "heart of the song" are talking about the psychological, psychological will, imagination, thinking and singing on the important and from the psychological point of view, to explain the deep tone of singing, song interpretation, singing, singing activities, singing emotions, singing state of psychological response and role, to promote the further development of vocal music psychology.

The Chinese vocal art psychology has made great progress, largely in the Western vocal introduction after China began. After decades of theoretical research, China's vocal art psychology has formed a more scientific theoretical system, and achieved some success. Such as: Zou Changhai's "singing psychology", "vocal art psychology", $\mathrm{Xu}$ Xing of the "imagination imagination" and other monographs, which has a greater impact.

\section{The State Analysis and Exploration of Vocal Music Performing Psychology}

At present, the research on the psychology of vocal music at home and abroad has achieved some achievements, but it is still at the initial stage. The content and scope of the study are often confined to the macroscopic aspects of vocal education psychology, vocal learning psychology and vocal performance psychology. There are more or less deficiencies and superficial discussion in the development of the research level, the depth and breadth of various aspects of research and other disciplines on the impact of vocal art psychology, etc. Furthermore, the vocal art performance psychological aspects of the brilliant study is involved in very little. At the same time, some vocal artists and scholars in the specific elaboration of vocal art psychology and its branch performance psychology, are difficult to find in addition to psychological reactions, will, attention, imagination and association, emotional and emotional, ability and thinking principles outside the point of view to study in depth. Such as vocal music art psychology research expert Zou Changhai, when he discussed the singing performance psychology which only grasp five aspects including the song's context and emotions, tone and syntax, singing art processing, performance and temperament, and performance changes. Both in terms of the depth, breadth, or the content and form of the discourse, it lacks the strong and theoretical significance of the theory and practice, and the comprehensiveness, objectivity and scientificity of the research are to be further demonstrated. At least, the research on the influence of the properties, essential characteristics and related disciplines of the vocal music art is somewhat incomplete and regrettable.

Look from the vocal performance psychological attributes, ethnic characteristics, life characteristics and time characteristics, shown in Figure 1, are still accompanied by the development of vocal music art, and become the inherent attributes and features.

Ethnic characteristics is a natural style, form, the combination of the ideological content, and is a nation formed long-term characteristics of tradition and custom, which has rich profound historical culture connotation. This is a strong proof and embodiment in vocal music art, such as the Chinese Tibetan style, Hunan folk style, the German Bavarian style, the British Irish style, the western United States folk songs and other folk songs, which is the formation of historical and national portrayal and reaction. The vitality of the vocal art and the source of the material and the soul are from the human life, which is the form of indirect reaction of human life and determined by the nature and aesthetic attributes of vocal music. Leaving the life of art, it is tantamount to passive water, without the wood, without its existence value and meaning. Therefore, the development of vocal art must be with experience life, from life to obtain vivid, rich material. Vocal music performance research also needs to seize the characteristics and vitality of life, combine with human psychological attributes, double steps, comprehensive, scientific and rich vocal art. The characteristics of vocal music in the era of vocal music is particularly evident. Different periods, people's ideological content are marked with the imprint of the times, vocal art became the symbol of the times, such vocal art and performance psychology has also become the mainstream of the times. During the war of resistance against Japan, various anti-japanese folk songs of China sprang 
up, reflecting the hardships, confidence and national spirit of resistance against Japan. In the construction of the People's Republic of China, the sound style of the northern shaanxi folk songs constantly mobilized people to build a new home. The Times are developed, and the art has to keep pace with the Times, and only a trickle of active sources of supply will flourish. Anyhow, ethnic characteristics and life characteristics, time characteristics is one of the important properties of vocal music art and its branch, left the three characteristics, study vocal music art performance psychology are biased in theory and in practice. Whether from the physiological angle, or the psychological angle of the performance psychology, should pay enough attention to the three macro characteristics.

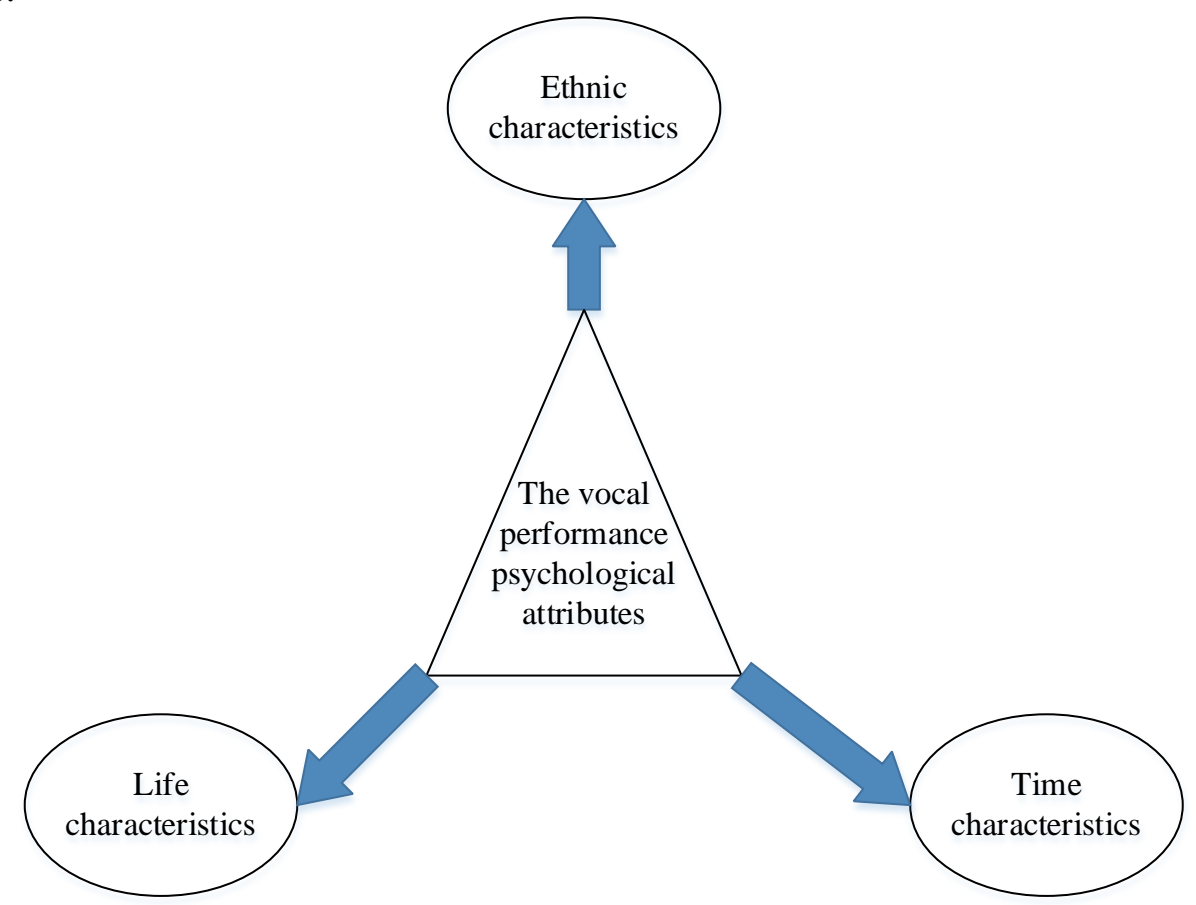

Figure 1. The vocal performance psychological attributes

The psychology of vocal performance is mainly manifested in the aspects of body language, singing, expression and sound environment, shown in Figure 2.

These three points are closely connected with artist inner psychological and interaction. The specific performance psychological research should start from these three points on the macro comprehensive rich vocal music aesthetics. The body language includes the individual's specific behavior and the overall form of the combination of hands, feet and facial behavior. One table is a combination of each other, one that drives each other, the natural one, and the perfect art form. "The way we sing, not only to study the law of the law of the voice, language, and to study the relationship between sound and language, research in different vocal style, type, how to according to the requirement of content and the artistic performance to coordinate and properly handle voice and the relationship between language, rhythm and language, the best way to make them to cooperation, unity in a strong artistic charm and aesthetic value of vocal music performance." This shows that the status of language is no longer unspoken. Singing and its expressions mainly discuss the psychology of vocal performance from the angle of expression and expression. Facial expressions are mainly the changes in the eyes, eyebrows, mouth and facial muscles. "The eyes are the window of the soul," and every kind of look, such as sadness, joy, excitement, sadness, fear and fear, contains rich and profound thoughts and feelings. That is to say, these eyes are mainly provided by psychological emotions and emotions, and what emotions and emotions naturally appear to match. The same is true of other emojis. Throughout the ages, almost every artist and performer has attached great importance to the relationship between expression and sound, and has accumulated a theoretical foundation. The function of sound environment to acting psychology is often ignored by others. In fact, it's a very important factor. Sound environment consists of the combination of music, the audience's emotional state, such as the atmosphere of the scene, the 
artistic charm of vocal music should be realized in the environment. Moreover, the singer's performance psychology changes fast, the influence of the environment is big, can cause complete preparation in a moment to fail the painful result of failure. However, the research theory about sound environment and performance psychology is not enough, and needs artists and scholars to enter more and more abundant blood, and the development of active theory.

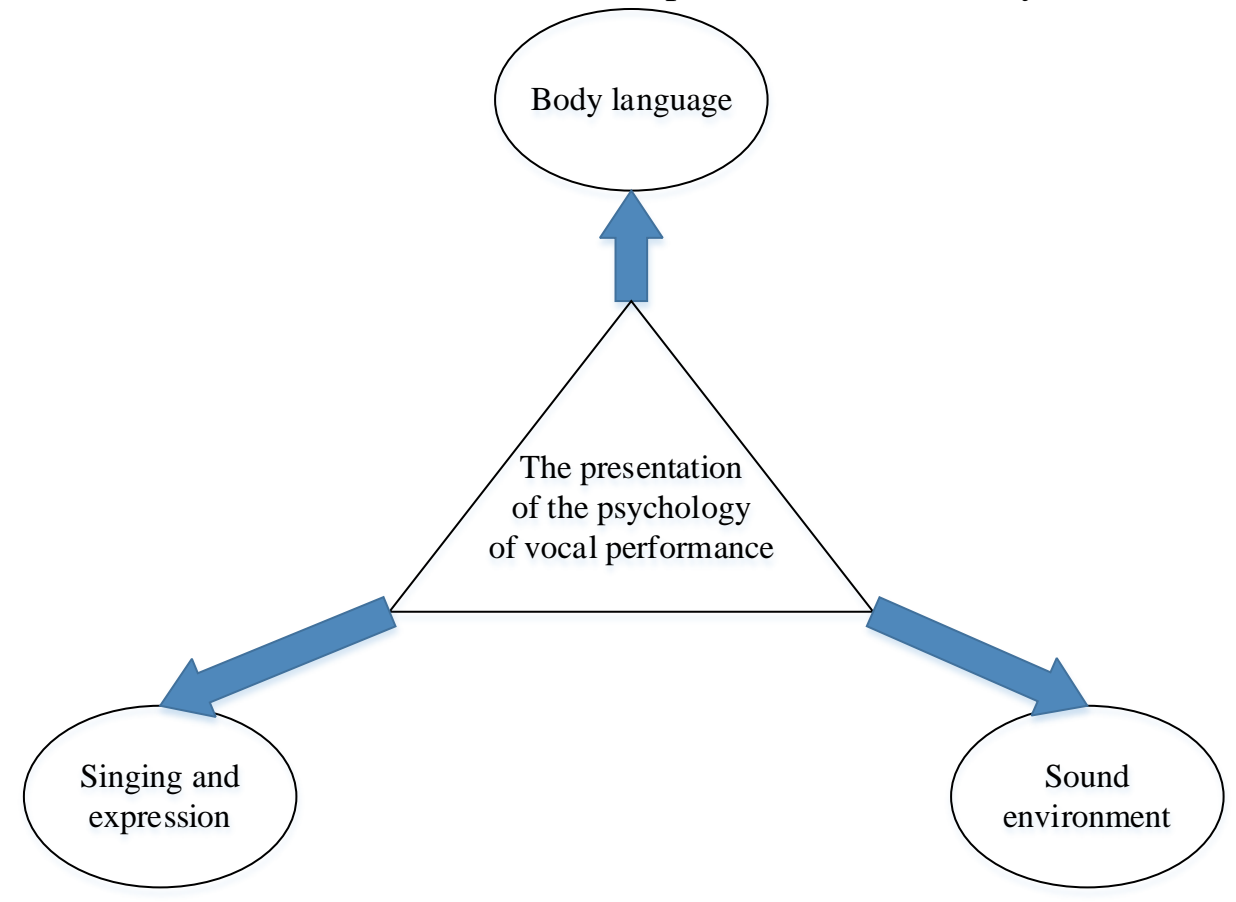

Figure 2. The presentation of the psychology of vocal performance

\section{The Research Trend of the Artistic Psychology of Vocal Music}

Based on years of research and observation, this paper will discuss the research attention and trends of vocal art performance psychology on the following aspects, shown in Figure 3.

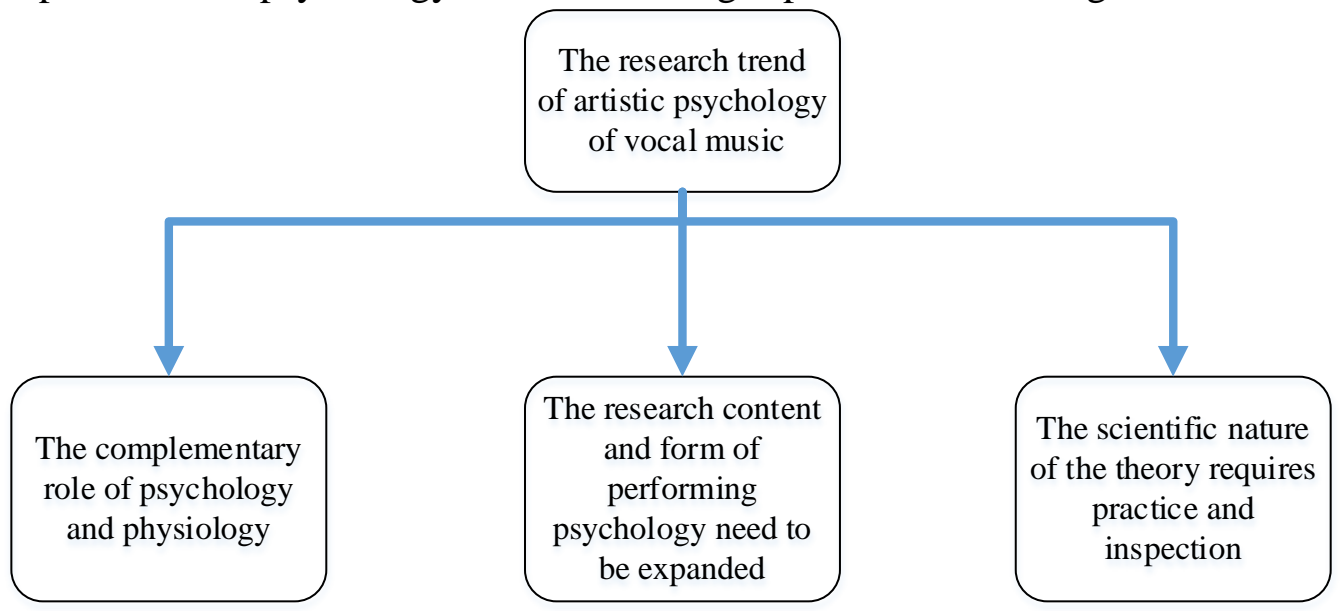

Figure 3. The research trend of artistic psychology of vocal music

The Complementary Role of Psychology and Physiology. The vocal music performance is both a technology and an art. The technology is the requirement of physiology, and art is the requirement of psychology. Lack of technology, the art of vocal performance is one-sided; Ignoring art, just the technological angle of prosperity is also a stumbling block to full awareness of sound. In the past, the study of vocal music has been too psychological or even serious. Psychology is now thriving in vocal music, growing fast but too blind. From the vocal music art law of development of observation, the ends of physiology and psychology on the same scale, balance and spot is the prosperity of vocal music art. The study of vocal music art performance cannot be confined to the 
psychological level, also need the balance point and point, this just accord with the law of the vocal music art performance psychology research, purpose and direction.

The Research Content and Form of Performing Psychology Need to be Expanded. As mentioned above, the research on the psychology of vocal music art and its branch performance psychology have a superficial side, mainly in content and form. In content, not only need to strengthen the depth of the research, the law of nature to explore, and to perform psychological research dimensions expand, even from the perspective of aesthetics, philosophy, literature and other disciplines to enrich and prosperity of vocal music art. In form, vocal performance is often limited to body language, expression, melody and external environment, such as expressing form of external embodiment and foil. On the one hand, the expression of mature forms needs to be further excavated the artistic charm and aesthetic culture. On the other hand, the form of the original form, the forms of diversity, richness and aesthetics are realized.

The Scientific Nature of the Theory Requires Practice and Inspection. Throughout the history of the development of vocal music art, it can be seen that the development of vocal music art no matter in practice, or in the theoretical research, has made remarkable achievements and unprecedented social status, the social life and spiritual civilization construction, culture construction has important influence. Outstanding achievements, however, is always accompanied by the emergence of the error theory, in the vocal music art highly developed at the same time. A lot of junk error theory with vocal music culture, has brought about many negative effects, even restrict the vocal music art, slow development and stagnation. Sound music requires scientific theory, and needs to be tested, perfected and prospering in practice. It is an important basis for enriching and prospering the social life and spiritual and cultural life of human beings, and it is necessary to pay attention to the theory in the future. At the same time, we should pay attention to the theory and practice.

\section{Conclusion}

This paper reviews the psychological history of vocal art performance and prospects its development trend. Music has a long history, the charm of music is fascinating, the life of music is vigorous. In vocal music art highly developed in today's world, how to promote further prosperity and development of vocal music in the service of mankind, is calling for more better vocal music artists and scholars devote themselves to the research and practice in this great cause.

\section{References}

[1] Jiang Shuxiang. The state of psychology in vocal music performance[J]. Journal of Bingtuan Education Institute, 2005, 4: 52-53.

[2] Liu Bo. About vocal music performing arts in psychological quality training[J]. Journal of Yangtze University (Social Sciences), 2007, 30(2): 111-112.

[3] Zhang Shenghuan. The artistic quality of vocal music is performed from the national vocal music [J]. Journal of Tonghua Normal University, 2011, 32(9): 59-62.

[4] Yu Hui. The psychological impact of vocal music[J]. Liaoning Higher Vocational Technical Institute, 2008, 10(12): 94-96.

[5] Zhou Jiequn. Effect of psychological activity on singing techniques[J]. Journal of Huaihai Institute of Technology, 2011, 9(12): 97-99.

[6] Chai Guangyu. The study of vocal music from the psychological aspect[J]. Journal of Xinzhou Teachers University, 2006, 22(3): 48-50. 